\title{
Responsabilidad social corporativa, actividades empresariales y desarrollo sustentable Modelo matemático de las decisiones en la empresa
}

\author{
Elvio Accinelli Gamba \\ Facultad de Economía, \\ Universidad Autónoma de San \\ Luis Potosí \\ elvio.accinelli@eco.uaslp.mx
}

\section{José Luis de la Fuente}

García

Facultad de Economía, Universidad Autónoma de San Luis Potosí

joseluis.delafuente@uaslp.mx

\section{Resumen}

En este trabajo se realiza una revisión de la literatura relacionada con la responsabilidad social corporativa, enfatizando su relación con el desarrollo sustentable, su descripción y evolución; asimismo, se presentan distintas reflexiones con respecto a la actuación que pudiera esperarse de las empresas en torno de esta situación, la cual se plantea a través de un modelo matemático, en el que - utilizando una matriz- se representan las distintas opciones que puede tomar la empresa. A manera de conclusión, podemos comentar que bajo determinadas condiciones sociales y técnicas las empresas optarán por una política de responsabilidad social corporativa sustentable como consecuencia del deterioro medioambiental que su propia acción conlleva. De igual manera, se muestra que en otro caso es posible para una autoridad central diseñar una política de incentivos capaz de lograr que las empresas opten por una política sustentable, aun cuando el factor de descuento futuro sea muy alto.

Palabras clave: desarrollo sustentable, responsabilidad social corporativa, gestión organizacional, toma de decisiones. 


\title{
Corporate social responsibility, business activities and sustainable development. Mathematical model of business decisions
}

\begin{abstract}
In this paper we review the literature related to corporate social responsibility, emphasizing its relationship to sustainable development, description and evolution, as well as different thoughts regarding the actions that might be expected from companies in relation to corporate social responsibility, a situation that is presented through a mathematical model, introduced by using a matrix that allows us to present the different ways in which a company can act to address this kind of decisions. We conclude that, under some social and technical conditions, companies will choose a sustainable corporate social responsibility policy, as a consequence of the environmental damage of their own operations. It is also shown, that it is possible for a central authority to design an incentives policy that can make companies choose a sustainable policy, even if the discount rate is high.
\end{abstract}

Keywords: sustainable development, corporate social responsibility, organizational management, decision making.

\section{Introducción}

En los últimos años hemos sido testigos de cómo el medioambiente se ha ido colocando como un aspecto central en las agendas políticas, empresariales y sociales, ya que las consecuencias de la llamada problemática medioambiental representan un reto a escala global, por lo que la respuesta a esta situación implica a todo el mundo y no sólo a unos cuantos países y empresas.

Lo anterior ha tenido como consecuencia el desarrollo de un entorno social, político y económico incierto y cambiante en el ámbito empresarial porque la aparición de nuevas preocupaciones por parte de la sociedad, así como de nuevas legislaciones relacionadas con el cuidado del entorno natural, han supuesto una serie de obstáculos en el desarrollo de los procesos productivos de muchas organizaciones; todo ello resulta en cambios de las formas de producción, los materiales y los energéticos utilizados en dichos procesos; además, en la forma de tratar y disponer los residuos resultantes de los mismos.

Actualmente, los expertos mundiales en los temas de medioambiente y cambio climático presentan un panorama global muy desolador: algunos argumentan que si 
no se actúa y se reduce el ritmo y la cantidad de emisiones de contaminantes que se depositan en el entorno natural, estaríamos muy cerca de llegar a lo que se denomina punto de no retorno; es decir, un punto en el que el medio ambiente no lograría recuperarse, incluso deteniendo toda actividad generadora de impacto en él.

Es por esto que resulta importante poder plantear cuáles son las opciones que puede tener una empresa en relación con la decisión de implementar una política sustentable en el desarrollo de sus actividades, de forma que se respete el principio económico de maximización del beneficio.

Nuestro trabajo se enfoca en presentar, a través de un modelo matemático plasmado en una matriz, las distintas opciones que tiene la empresa para operar en relación con el medio ambiente; es decir, si optar por la maximización de un beneficio financiero o de un beneficio ambiental, o bien de un justo equilibrio entre estas dos posturas; todo desde una perspectiva racional de maximización del beneficio. El resto se estructura de la siguiente manera: primero se presenta una revisión de la literatura relacionada con la actuación empresarial y la problemática medioambiental; después se describe nuestro modelo matemático y la matriz de opciones que tienen las empresas para la adopción de una política medioambiental sustentable que permita la maximización del beneficio; por último, se presentan las conclusiones de este trabajo.

\section{La problemática medioambiental y la actuación empresarial}

En las últimas décadas del siglo pasado y en los primeros años del actual, hemos sido testigos de una serie de cambios en el sistema de valores del entorno social y político en el que se desarrolla la vida empresarial. La preocupación por la interacción que la empresa tiene con su entorno natural se ha convertido en un aspecto por considerar por parte de los directivos de las mismas.

Lo anterior se originó a partir de las presiones sociales que tuvieron lugar desde mediados del siglo pasado. En la literatura se señalan distintas cumbres mundiales y diversos acontecimientos que marcaron un antes y un después con la forma de ver el entorno natural; así, algo que se consideraba inagotable ahora parece tener una fecha de caducidad, lo que puede llegar a representar una posible restricción de acceso a materias primas y energéticos para la actividad de las empresas. 
En 1972, con la publicación del informe Meadows "Los límites del crecimiento", se activa una cierta conciencia sobre la relación que mantenía el crecimiento incontrolado, tanto industrial como demográfico, y las limitaciones medioambientales para hacer frente a dicho crecimiento.

Los aspectos medioambientales se consolidaron como un problema de escala global en los años ochenta y principios de los años noventa. En este sentido, la Comisión Mundial para el Medio Ambiente y Desarrollo, cuyo propósito era el de proponer estrategias de actuación medioambiental a corto plazo que permitieran alcanzar el desarrollo sustentable para el año 2000 y posteriores (Aitken, 1989), elabora en 1987 el informe Brundtland "Nuestro futuro común", en el que se defiende un crecimiento de la producción y el consumo diferente al utilizado hasta ese momento, así como la aparición del concepto de desarrollo sustentable.

El informe Brundtland (UNWCED, 1987) fue la culminación de un proceso largo que examinó las interacciones humanas con el medioambiente; de esta forma y de acuerdo con el mismo, se afirma que es sustentable aquel desarrollo que permite la satisfacción de las necesidades de la humanidad sin poner en riesgo la satisfacción de las necesidades de las generaciones futuras (Larrinaga, 2002).

En 1992 se celebró en Río de Janeiro la convención de las Naciones Unidas sobre medio ambiente y desarrollo; en ella los líderes del mundo firmaron la denominada Declaración de Río, que propone un marco de actuación basado en el concepto de desarrollo sustentable (Moneva y Llena, 2006). De esta forma, dicho concepto ocupa un lugar prioritario en el planteamiento de la pregunta ¿qué tipo de sistema económico puede permitir que se cumplan las necesidades de todos, de una forma medioambientalmente sustentable y socialmente justa?

La pregunta anterior deja ver que el concepto de desarrollo sustentable engloba cuestiones que van más allá de las ambientales, ya que éste comprende los principios de justicia intrageneracional (generaciones presentes) e intergeneracionales (generaciones futuras) (Gray y Bebbington, 2000; Larrinaga, 2002), de forma que el modelo económico que se quiera plantear debe tener en cuenta la perspectiva del largo plazo y no sólo pensar en soluciones paliativas en el corto plazo, pues un desarrollo sustentable debe garantizar que las generaciones futuras tendrán las mismas oportunidades de acceso a los recursos naturales que las presentes. 
Es por esto que Bebbington (2001) argumenta que la pregunta estructural inherente al desarrollo sustentable es aparentemente la de cómo organizar el sistema económico de manera que el desarrollo tome lugar sin dañar el medio ambiente en el que se basa el desarrollo presente y futuro. Por lo anterior es que el autor argumenta que existe una percepción de que se ha igualado el concepto de desarrollo sustentable con el de buena gestión empresarial, pero ambas no representan lo mismo.

Toda esta agitación que se presenta en el entorno empresarial ha tenido como consecuencia la aparición de una gran cantidad de normativa en la materia y de códigos de conducta vinculados a una aparente preocupación por el estado de deterioro que guarda el entorno natural; asimismo, y desde la perspectiva de una respuesta empresarial al problema, la aparición en las organizaciones de los sistemas de gestión medioambiental y las auditorías medioambientales (Fernández de Gatta, 2004). Sin embargo, dichas herramientas no siempre han ido acompañadas de los respectivos cambios en los sistemas informativos de la empresa y en la corriente general de gestión, por lo que permanecen como una parte de la estructura organizacional, pero de forma periférica al núcleo de toma de decisiones.

Diversos autores (Hernández, 1999; Garcés, 2006) argumentan la existencia de un aparente acuerdo en la literatura con respecto a la existencia de presiones que el entorno organizacional está ejerciendo sobre las empresas para que adopten una serie de soluciones dirigidas a la protección y conservación del entorno natural, ya sea a través de la legislación existente o a través de los mecanismos de mercado, por lo que resulta cada vez más común encontrar empresas que se presentan como socialmente responsables y medioambientalmente conscientes del impacto que su actividad tiene en el entorno natural, utilizando para esto la mercadotecnia, sus comunicados o sus informes anuales.

Este incremento por parte de los gobiernos en el interés, en las políticas de mercado y en la sociedad en general, sobre los impactos medioambientales de la actividad empresarial han repercutido para que distintas agrupaciones y gobiernos fomenten la aparición de códigos de conducta y de gestión empresarial; así, esas preocupaciones globales o de nivel macro buscan motivar la incorporación de estos aspectos de cuidado medioambiental en las empresas, o en un nivel micro (Banerjee, 2001; Crespo, 2002) de forma que poco a poco se vaya esparciendo entre los núcleos de gestión empresarial, y en todos los niveles de la misma, la cultura de sustentabilidad medioambiental. 
De esta forma se consolida el concepto de responsabilidad social corporativa (RSC) que aparece a mediados del siglo pasado y que busca colocarse como una forma de pensar la gestión empresarial basada en tres pilares: el económico, el social y el medioambiental; con este concepto las empresas deben ir más allá de sus obligaciones legales, en materia social y medioambiental, de forma que ayuden al crecimiento y el desarrollo del entorno en el que operan, así como a la conservación del medioambiente. En consecuencia, la RSC busca que las empresas no sólo entreguen beneficios económicos a los accionistas de las empresas, sino también busca que generen beneficios sociales y medioambientales (Vaca et al., 2007)

Uno de los problemas que presenta la RSC, así como la mayoría de los temas que se relacionan con el desarrollo sustentable, es la falta de un criterio único para definir y estudiar estos conceptos, ya que, por ejemplo, el caso del término de RSC ha sido definido de muchas formas (ver cuadro 1); todas esas definiciones son válidas porque la noción que subyace en el concepto es la misma en todas, puesto que van encaminadas a tratar de proponer una forma distinta de actuación y gestión empresarial.

Siguiendo esta idea, Castellanos et al. (2007) argumentan que de todas las definiciones de RSC podemos destacar un rasgo fundamental: supone un compromiso voluntario de las organizaciones de forma que con su actividad se genera un valor añadido para la sociedad, con lo que así supera la visión restringida de la consecución del máximo beneficio económico. 


\section{Cuadro 1 \\ Definiciones del concepto de Responsabilidad Social Corporativa (RSC)}

\begin{tabular}{|c|c|}
\hline Autor & Definición \\
\hline Carroll (1979) & $\begin{array}{l}\text { La responsabilidad social de las empresas engloba las expectativas } \\
\text { económicas, legales y éticas que la sociedad tiene de las } \\
\text { organizaciones (p. 500). }\end{array}$ \\
\hline $\begin{array}{l}\text { Libro verde: fomentar un } \\
\text { marco europeo para la } \\
\text { responsabilidad social de las } \\
\text { empresas (Comisión de las } \\
\text { Comunidades Europeas, 2001) }\end{array}$ & $\begin{array}{l}\text { La RSC es la integración voluntaria por parte de las empresas de } \\
\text { las preocupaciones sociales y medioambientales en sus operaciones } \\
\text { comerciales y sus relaciones con sus interlocutores (p. 7). }\end{array}$ \\
\hline Amnistia Internacional (2002) & $\begin{array}{l}\text { La RSC es el reconocimiento e integración en sus operaciones por parte } \\
\text { de las empresas, de las preocupaciones sociales y medioambientales, } \\
\text { dando lugar a prácticas empresariales que satisfagan y configuren sus } \\
\text { relaciones con sus interlocutores (p. 41). }\end{array}$ \\
\hline $\begin{array}{l}\text { World Business Council for } \\
\text { Sustainable Development } \\
\text { (WBCSD) (Fundación } \\
\text { Entorno, 2003) }\end{array}$ & $\begin{array}{l}\text { La RSC es el compromiso de la empresa de contribuir al desarrollo } \\
\text { económico sustentable. Trabajando con los empleados, sus } \\
\text { familias, la comunidad local, y la sociedad en general para mejorar } \\
\text { la calidad de vida (p. 114). }\end{array}$ \\
\hline $\begin{array}{l}\text { AECA } \\
\text { (Asociación Española } \\
\text { de Contabilidad y } \\
\text { Administración de Empresas, } \\
\text { 2004) }\end{array}$ & $\begin{array}{l}\text { La RSC es el compromiso voluntario de las empresas con el } \\
\text { desarrollo de la sociedad en la preservación del medio ambiente, } \\
\text { desde su composición social y un comportamiento responsable hacia } \\
\text { las personas y grupos sociales con quienes interactúa (p. 21). }\end{array}$ \\
\hline Fernández de Gatta (2004) & $\begin{array}{l}\text { La RSC se refiere al proceso mediante el cual las empresas deciden } \\
\text { voluntariamente contribuir al logro de una sociedad mejor y de un } \\
\text { medio ambiente más limpio (p. } 27 \text { ). }\end{array}$ \\
\hline Nieto (2004) & $\begin{array}{l}\text { La RSC es un concepto por el cual las empresas incorporan } \\
\text { voluntariamente criterios sociales y medioambientales en las } \\
\text { actividades económicas y en las relaciones con sus grupos de } \\
\text { interés (p. 253). }\end{array}$ \\
\hline Gallizo (2006) & $\begin{array}{l}\text { La RSC se refiere a la puesta en práctica de políticas formales y de } \\
\text { sistemas de gestión en el campo social y medioambiental, de las } \\
\text { que luego se debe informar de modo transparente y con contraste } \\
\text { externo de los resultados (p. 11). }\end{array}$ \\
\hline $\begin{array}{l}\text { Castellanos et al. } \\
\text { (2007) }\end{array}$ & $\begin{array}{l}\text { La RSC supone un reconocimiento e integración en la gestión } \\
\text { y las operaciones de las organizaciones, de las preocupaciones } \\
\text { sociales, laborales y medioambientales, y de respeto a los derechos } \\
\text { humanos, que den lugar a la elaboración de políticas, estrategias y } \\
\text { procedimientos que satisfagan a los diferentes grupos de interés y } \\
\text { que permitan configurar sus relaciones con éstos (p. 2622). }\end{array}$ \\
\hline
\end{tabular}


Las definiciones que se presentan en el cuadro 1 nos permiten apreciar, por un lado, la falta de consenso con respecto a lo que representa el concepto de RSC, como ya lo mencionábamos; pero también dejan ver, por otro, que la actuación empresarial en materia de medioambiente está siendo sujeto de examen tanto por la sociedad como por los gobiernos al hacer referencia a terceras partes interesadas (o stakeholders ${ }^{1}$ ). Recordemos que es del entorno externo a la organización de donde emanan todas estas propuestas de códigos de conducta y de responsabilidad ${ }^{2}$, por lo que la existencia de los stakehoders es lo que hace suponer que las empresas deberán tomar cartas con relación a su actuación en la materia.

De los conceptos presentados de RSC podemos realizar también una serie de reflexiones con respecto a lo que ésta supone para las empresas, que algunos autores (Hoffmeister et al., 2005; Nieto, 2005; Giraldo et al., 2006) consideran como las características de las políticas de RSC:

- Se trata de una adopción voluntaria que va más allá de las obligaciones jurídicas.

- La RSC está intrínsecamente vinculada al concepto de desarrollo sustentable porque las empresas deben orientar sus operaciones para favorecer el crecimiento económico, al mismo tiempo que garantizan la protección del medio ambiente y respeto social, incluidos los derechos humanos y los intereses de los consumidores.

- La RSC no es algo que pueda añadirse opcionalmente a las actividades principales de la empresa, sino que afecta a su propia estrategia y gestión, por lo que requiere de los instrumentos adecuados para ello.

\footnotetext{
'Estos terceros interesados, o grupos de interés, son denominados por algunos autores con el término stakeholder. En este sentido, la Asociación Española de Contabilidad y Administracion de Empresas (2004) define a los mismos como "aquellos grupos sociales e individuos afectados de una forma u otra por la existencia y acción de la empresa, con un interés legítimo, directo o indirecto, por la marcha de ésta. Qué influyen a su vez en la consecución de sus objetivos marcados y a su supervivencia".

${ }^{2}$ Algunas de estas propuestas son mencionadas por Fernández (2005) las cuales, a manera ilustrativa, son el pacto mundial de las Naciones Unidas (UN global compact), las líneas directrices de la OCDE, el Libro verde: Fomentar un marco europeo para la responsabilidad social de las empresas de la comisión europea, los principios Sullivan de RSC, la Global Reporting Initiative (GRI), o el AA 1000 Assurance Estándar (Account Ability), entre otras. Asimismo, Nieto y Fernández (2004) mencionan que las iniciativas pueden ser de dos tipos: normas de principios generales, como UN global compact o el Libro verde, y normas de aplicación, como lo sería el GRI y el Account Ability.
} 
- La RSC engloba un conjunto de acciones de formalización de sistemas de gestión, de comunicación, de transparencia informativa, desarrolladas en una dimensión tanto interna como externa a la empresa.

- La RSC transforma la fórmula básica de gobierno de la empresa, centrada en la relación bilateral entre accionistas y directivos (enfoque shareholder), en otra multilateral en la que participan todos los grupos de interés (stakeholders).

- La RSC modifica el proceso de toma de decisiones al añadir a los criterios de eficiencia económica la consideración del impacto ambiental y social de las operaciones de la empresa.

- Cuando las empresas informan que adoptan criterios RSC están comunicando a los grupos de interés que han decidido ajustar su comportamiento no sólo a lo establecido por los preceptos legales y los principios económicos, sino también a las normas, valores y expectativas sociales que dominan en su entorno.

Como se puede apreciar, la incorporación de criterios de RSC en la empresa implica todo un proceso de cambio; en ocasiones se requiere no sólo el replanteamiento de sus procesos productivos, sino que también puede llegar a ser necesario un replanteamiento de los objetivos y estrategias empresariales. Algunos autores argumentan que si los objetivos de un comportamiento socialmente responsable se incorporaran en los objetivos, estrategias de gestión y sistemas de gestión de la empresa, se enfatizaría su despliegue a toda la organización (Moreno, 2004; Lizcano y Nieto, 2006).

Los beneficios que la empresa puede obtener por la adopción de este tipo de comportamientos medioambientalmente sustentables son, de acuerdo con De Lara (2003), entre otros:

- Reducción de riesgos derivados de ciertos comportamientos socialmente irresponsables.

- Puede ser un indicador de buena calidad en la gestión.

- Reducción de costos.

- Nuevas oportunidades de negocio.

- Desarrollo de ciertos activos intangibles: reputación, imagen de marca e imagen pública, etcétera. 
Lo expuesto hasta este momento nos permite plantear la encrucijada que supone para la empresa el posicionamiento del entorno natural como una variable que puede afectar el desarrollo de las actividades empresariales. Es decir, por un lado, nos encontramos con distintos actores del entorno externo a la organización que reclaman un comportamiento más responsable con respecto al uso que se hace de los recursos naturales en las actividades de las empresas, lo que se ha manifestado con la aparición de nueva normativa medioambiental, códigos de conducta como la RSC, sanciones más estrictas, entre otros aspectos; por otro, tenemos a los accionistas y propietarios de las empresas, los cuales demandan a sus directivos maximizar, en el menor plazo posible, los beneficios derivados de su inversión (Porter y Kramer, 2003), lo que lleva a una mayor producción y un mayor consumo de recursos naturales, de forma tal que desde esta óptica existe una diferencia de implementación operativa entre lo que demanda el entorno externo a la empresa y lo que exige el entorno interno de la misma.

Siguiendo esta idea, Gray y Bebbington (2000) sugieren que la evidencia que nos muestra la investigación en estos temas es que existe un conflicto entre los objetivos corporativos y el atractivo de la sustentabilidad. Al parecer un buen desempeño medioambiental o un comportamiento socialmente responsable con el entorno natural por parte de las empresas está en conflicto con el objetivo central de toda empresa: la maximización del beneficio. Se argumenta en la literatura que el desempeño ambiental ha sido visto en términos de los impactos generados en el entorno natural por el desarrollo de las actividades de la empresa (Henri y Journeault, 2009); por lo tanto, mientras los retos medioambientales sigan siendo más complejos y los costos asociados a la reducción de esos impactos continúen elevándose, la solución a ese conflicto de intereses pareciera no estar cercana, ya que como mencionan Walley y Whitehead (1994) para todos los aspectos medioambientales el valor para el accionista es la única medida que vincula y no el cumplimiento, las emisiones o los costos.

Por esta razón, y bajo los argumentos expuestos en estos últimos párrafos, nuestro trabajo cobra relevancia, ya que desde nuestra perspectiva de estudio, y de acuerdo con Al-Tuwaijri et al. (2004), la pregunta central que siempre se plantea es si el ser ecológico es bueno para las ganancias, por lo que poder plantear el hecho de tomar una decisión racional con respecto a la adopción de una política sustentable en las operaciones de la empresa cobra relevancia. 
La pregunta anterior se enmarca dentro de las investigaciones que defienden el paradigma de ganar-ganar ${ }^{3}$ donde, de acuerdo con Gray y Bebbington (2000), se aborda la cuestión sobre cómo pueden utilizarse herramientas de gestión, información y control organizacionales para identificar ahorros financieros en, por ejemplo, el uso de recursos materiales, desperdicios de energía, emisiones; así, al lograr identificar y reducir esos desperdicios, la consecuencia lógica será la disminución del impacto medioambiental de la organización.

\section{La adopción de una política medioambiental sustentable: un modelo de toma de decisiones}

El paradigma ganar-ganar apoya la idea de que es posible una reducción del impacto medioambiental de la empresa sin dañar su posición financiera; incluso defiende que no sólo no dañaría dicha posición, sino que, al identificar esas ineficiencias en el manejo de recursos, una gestión y un adecuado control de los mismos contribuiría a mejorar la posición financiera de la organización.

Para ilustrar mejor este paradigma, así como los panoramas adicionales que pueden existir, en la figura 1 se presentan en forma de matriz los supuestos del modelo matemático que hemos desarrollado para ilustrar las opciones que tiene la empresa al momento de tomar la decisión de adoptar una política medioambiental sustentable.

\section{Figura1}

\section{Matriz de opciones de la empresa}

\begin{tabular}{|c|c|c|c|}
\hline \multirow[t]{2}{*}{ Perder } & & & $\longrightarrow$ Ganar \\
\hline & & \multicolumn{2}{|c|}{ Posición financiera } \\
\hline \multirow{2}{*}{$\underset{\text { Ganar }}{\downarrow}$} & \multirow{2}{*}{ Medio ambiente } & $\operatorname{Pma}(t), P^{1} p f(t)$ & $P^{\prime} m a(t), G^{1} p f(t)$ \\
\hline & & $G^{\prime} m a(t), P^{2} p f(t)$ & $G^{\prime} m a(t), G^{2} p f(t)$ \\
\hline
\end{tabular}

El primer recuadro corresponde a una empresa ineficiente, pues produce con pérdidas financieras $P^{1} p f(t)$. Por otra parte, la propia ineficiencia de la empresa la lleva

${ }^{3}$ El paradigma ganar-ganar aparece en el libro de los siete hábitos de la gente altamente efectiva (Covey, 2003), en el cual uno de los hábitos propone el desarrollo de fórmulas de negocio bajo este paradigma, en el cual la empresa gane y el cliente también, ya que si no se respeta esta igualdad, las relaciones comerciales pudieran no perdurar en el futuro, como lo sería en el caso de ganar-perder, situación en la que la empresa gana y el cliente pierde, por lo que el riesgo en que se incurre es que el cliente no regrese con la empresa o viceversa. 
a contaminar el medio ambiente produciéndose un deterioro en la calidad de éste que se mide por $P^{\prime} m a(t)$. El recuadro de abajo a la izquierda corresponde a una empresa también ineficiente por haber elegido una política altamente cuidadosa con el medio ambiente, que produce un beneficio medioambiental que se mide por $G^{\prime} m a(t)$, pero con pérdidas financieras $P^{2} p f(t)$. Obviamente estas empresas o cambian su política de producción o no podrán mantenerse en el mercado en el largo plazo.

Los recuadros de la derecha corresponden, respectivamente, a una empresa que produce de forma eficiente; en el caso de arriba sin considerar los daños al medio ambiente y en el caso de abajo cuidándolo, ambos casos con beneficios positivos. Mientras que $G^{1} p f(t)$ corresponde al caso de la empresa que produce con deterioro del medio ambiente, representado por $P^{\prime} m a(t), G^{2} p f(t)$ corresponde a las ganancias de la firma que elige preservar el medio ambiente, representado por $G m a(t)$, aun a costa de una ganancia menor en el periodo $t$ dado que $G^{1} p f(t)>G^{2} p f(t)$. Llamaremos a estas políticas posibles de la empresa o planes de producción 1 y 2 , respectivamente.

Este esquema se reproduce periodo tras periodo, por lo que las firmas ineficientes se retiran del mercado y las eficientes deberán elegir su política, que repercute en el siguiente periodo.

Debe considerarse que si la forma eficiente, que es la que se mantiene, elige obtener en el periodo altos beneficios sin cuidar el medio ambiente, los costos de producir en el siguiente periodo serán más altos, lo que da lugar a beneficios menores en el siguiente periodo. Definimos como:

$$
G^{i} p f(t / j),(i=1,2, \quad j=1,2)
$$

Las ganancias de la firma eficiente en el periodo t que elije la política i, habiendo elegido $\mathrm{j}$ en el periodo anterior.

En este sentido, es conveniente recordar lo mencionado por Porter y Van der Linde (1995 a y b) en relación con que toda forma de contaminación es un desperdicio económico; es decir, cuando la chatarra, sustancias dañinas, o formas de energía son liberadas en el entorno como contaminación es una señal de que los recursos han sido utilizados incompleta, ineficiente e ineficazmente. Asimismo, estos auto- 
res señalan que las empresas deberán realizar actividades adicionales a la operativa normal de la organización como el manejo, almacenaje y disposición de los residuos; estas actividades añaden un costo adicional y no crean ningún valor para los clientes y los accionistas.

Asumimos que las únicas condiciones económicas que se modifican entre el período $\mathrm{t}$ y $\mathrm{t}+1$ son las referidas al medio ambiente y que éste depende exclusivamente de la política seguida por las firmas, mientras que la demanda, la oferta y la tecnología disponible se mantienen iguales en ambos periodos. De esta forma, los beneficios obtenidos en un periodo y otro dependerán exclusivamente de la política medioambiental elegida por las firmas. Asumimos que una política elegida en el período t, que no tome cuidado del medio ambiente, ocasiona costos adicionales en el periodo $t+1$. La repercusión de la política elegida en el período t en la actualidad, $\mathrm{t}+1$, se resume en las siguientes desigualdades:

Mientras que:

$$
G^{2} p f(t+1 / 1)<G^{1} p f(t+1 / 1)<G^{1} f(t)
$$

$$
G^{1} p f(t+1 / 2)<G^{2} p f(t+1 / 2)<G^{2} f(t)
$$

La primera desigualdad refleja el hecho de que las ganancias en el periodo $t+$ 1 , correspondientes a una firma que produjo en el periodo t con deterioro de la calidad ambiental $(\mathrm{i}=1)$, serán menores que las obtenidas en el periodo anterior, independientemente de la política que siga en $\mathrm{t}+1$.

La segunda desigualdad refleja el hecho de que las ganancias por obtener en el periodo $t+1$ por una firma, que en el periodo $t$ haya optado por producir sin contaminar ( $\mathrm{i}=2$ ), no serán menores que las obtenidas en el periodo anterior, independientemente de la política que elija seguir en $\mathrm{t}+1$.

Ahora bien, supongamos que la empresa mira sus beneficios atendiendo al periodo actual $t$ al siguiente $(t+1)$. La desigualdad es:

$$
\begin{aligned}
& G^{1} p f(t+1 / 1) e^{-r}+G^{1} p f(t)<G^{2} f(t+1 / 2) e^{-r}+ \\
& G^{2} p f(t)<G^{1} f(t+1 / 2) e^{-r}+G^{2} p f(t)
\end{aligned}
$$


Lo anterior hace referencia al hecho de que la firma preferirá producir en el primer periodo sin contaminar, pues sus beneficios agregados, aun en el caso de modificar su política para el periodo siguiente, serán mayores que si hubiese optado por contaminar en el primer periodo, aunque se verifique la desigualdad $G^{1} p f(t)>G^{2} p f(t)$. No obstante, si la producción termina en el periodo $t+1$, la firma sin ser contaminante en el periodo t elegirá contaminar en el periodo $t+1$. Pero si hay un siguiente $(t+2)$, la firma preferirá elegir no contaminar también en el periodo $t+1$.

El parámetro r que aparece en la exponencial representa la tasa de descuento intertemporal. Se puede asegurar que la firma producirá atendiendo a la preservación del medio ambiente, si la tasa de descuento no es muy alta; esto es:

$r<-\log \left[G^{1} p f(t+1 / 2)-G^{1} p f(t+1 / 1)-\right] /\left[G^{1} p f(t)-G^{2} p f(t)\right]$

Debe tenerse en cuenta que la tasa de descuento intertemporal hace referencia al valor actual que un agente económico otorga a una unidad de beneficio obtenida en el futuro, por lo que la desigualdad anterior supone que la firma no es absolutamente prescindible del futuro.

Con los supuestos indicados, es el propio deterioro ambiental, que repercute sobre los beneficios futuros, el que lleva a la empresa a elegir una política sustentable. El caso señalado corresponde a un control automático de la empresa que busca permanecer en el mercado y la sustentabilidad de la producción. Esto puede verse alterado por la existencia de empresas interesadas solamente en el muy corto plazo y en el beneficio inmediato, sin interés por el futuro.

Siguiendo con estas ideas, la empresa eficiente que trabaja sin cuidado del medio ambiente incurrirá en costos adicionales en el segundo periodo, los cuales denominaremos $C L$; ellos están asociados a la necesidad de limpieza del entorno debido a la suciedad ocasionada por la descuidada producción en el periodo anterior o debido a una tasa de contaminación impuesta por una autoridad central, capaz de monitorear la contaminación en forma precisa. De esta forma:

$$
G^{1} p f(t+1 / 1)=G^{1} p f(t)-C L
$$

Supongamos que los beneficios asociados a estas formas de producir se mantienen constantes a lo largo del tiempo. Consecuentemente la diferencia de los beneficios 
$\Delta=G^{1} p f(t)-G^{2} p f(t)>0$ se mantiene también constante. Los beneficios de la empresa contaminadora en el segundo periodo se verían reducidos en la cantidad $C L$, por lo que los beneficios presentes en $t=t_{0}$ serían iguales a:

$$
V^{1}\left(t_{0}\right)=G^{1} p f\left(t_{0}\right)+\left(G^{1} p f\left(t_{0}\right)-C L\right) e^{-r}
$$

Donde $r$ es la tasa de descuento intertemporal.

Los beneficios correspondientes a la empresa que produce no sólo de manera eficiente, sino también con cuidado del medio ambiente serán:

$$
V^{2}\left(t_{0}\right)=G^{2} p f\left(t_{0}\right)+G^{2} p f t\left({ }_{0}\right) e^{-r}
$$

Por lo que podría asegurarse la forma no contaminante de producir, si se verifica la desigualdad $V^{2}\left(t_{0}\right)-V^{1}\left(t_{0}\right)>0$; esto es:

$G^{2} p f\left(t_{0}\right)+G^{2} p f\left(t_{0}\right) e^{-r}>G^{1} p f\left(t_{0}\right)+\left(G^{1} p f\left(t_{0}\right)-C L\right) e^{-r}$

O equivalente si

$$
\Delta<C L \frac{1}{1+e^{r}}
$$

Por lo que la condición (8) asegura la producción sin contaminación, sin necesidad de una futura participación de alguna autoridad central.

En otro caso, si la tasa de descuento futuro verifica $r>\operatorname{In}\left(\frac{C L}{\Delta}-1\right)$, entonces debería intervenir la autoridad central aumentando el impuesto a la contaminación si éste ya existiese y fuera considerado en $C L$, o bien imponiendo uno nuevo para asegurar la producción futura sin contaminación. Nótese que un valor muy grande para $r$ significa que el futuro importa relativamente poco, por lo que el valor presente de los beneficios futuros es relativamente bajo. En este caso la actuación de la autoridad central se hará más necesaria a los efectos de asegurar la producción no contaminante.

Debe tenerse en cuenta que la participación de dicha autoridad genera costos adicionales, que implicarán a su vez la necesidad de incrementar el impuesto a la contaminación. Si el control por parte de la autoridad central no puede ser exhaustivo, deberían determinarse formas aleatorias de control; en este caso, el impuesto a la 
contaminación, que es necesario para asegurar la producción limpia y que puede llegar a ser excesivamente alto, puede traer como consecuencia el desarrollo de un comportamiento estratégico por parte de las firmas para burlar los controles.

Para reducir los costos de ejercer este control, la autoridad central podría implementar un control aleatorio de forma tal que en lugar de $C L$ la empresa debería descontar el valor esperado de este control; es decir, $E(C L)=\mathrm{p}(v) C L+(1-p(v)) 0$, donde $p(v)$ es la probabilidad de que la autoridad central visite a la empresa contaminante, atendiendo a que si la empresa contamina y no es monitoreada no tendrá cargo alguno por la contaminación ocasionada por ella. La condición para que se realice una producción no contaminante será entonces:

$$
p(v)>\frac{\Delta}{C L}\left(1+e^{r}\right)
$$

Implementar esta política será posible siempre que $\Delta\left(1+e^{r}\right)>C L$. En caso contrario se deberá imponer un impuesto incrementando $C L$, o bien apoyar políticas que reduzcan los costos de oportunidad de la conservación del medio ambiente.

Por lo expuesto en párrafos anteriores, las bondades de una política de preservación del medio ambiente pueden dar como resultado una reducción en los costos de producción al introducir una tecnología más cuidadosa con el entorno natural. Tal es el caso de la empresa 3M que implementó un programa de reducción de la contaminación denominado PPP (por sus siglas en inglés, Pollution Prevention Pays) con el que se lograron unos ahorros considerables (500 millones de dólares) (Walley y Whitehead 1994), o bien de la acción de organismos de control que toman medidas que afecten a los beneficios de firmas cuyos planes de producción no contemplan el cuidado del medio ambiente, ya que como explica nuestro modelo los costos asociados a la intervención de una autoridad central serían mayores.

De esta forma, y si partimos de la base de que un buen desempeño medioambiental estará marcado por la minimización de contaminantes, la conservación de los recursos y la reducción del desperdicio (Al-Tuwaijri et al., 2004), el vínculo entre la mejora en los resultados financieros y la mejora en el desempeño ambiental no parecen estar en un grado de conflicto, sino que, por el contrario, parecen ser parte de un proceso de mejora financiera a través de un uso más racional de los recursos en los procesos productivos, motivado por la adopción de una política empresarial sustentable. 


\section{Conclusiones}

Creemos que existen pocas dudas con respecto a la existencia de una relación entre el estado de deterioro que guarda el entorno natural y el incremento que en los últimos años ha tenido la actividad industrial y productiva en nuestro planeta; por ello, es de vital importancia un cambio de paradigmas en los modelos de gestión empresarial para comenzar a remediar esta situación.

En este sentido, la aparición de códigos de conducta enfocados al desarrollo de una responsabilidad social corporativa pueden ser considerados como una punta de lanza que busca que las empresas identifiquen oportunidades de mejora internas, ya sea en sus procesos productivos o en la forma en que desarrollan sus actividades, lo que derivará en el desarrollo de empresas que busquen implementar estrategias y modelos de negocios que permitan alcanzar una actitud ganar-ganar con respecto a la problemática medioambiental a través del desarrollo de políticas empresariales sustentables.

El modelo planteado nos permite apreciar cómo los resultados financieros de la empresa se ven afectados por el mismo daño al medio ambiente, por lo que si las empresas buscan prevalecer en el largo plazo, deben prestar atención a los procesos de producción que desarrollan para que puedan identificar tecnologías, programas, estrategias o políticas que permitan disminuir el deterioro del entorno natural, al mismo tiempo que se mejora y sostiene la posición financiera en el mediano y largo plazo.

Es decir, desde una perspectiva de maximización en la utilización de recursos, incluyendo aquellos subproductos o "desperdicios" derivados de un proceso productivo, el hecho de implementar y desarrollar políticas empresariales que busquen la conservación del entorno natural en el corto y mediano plazos van a facilitar que la empresa alcance una sostenibilidad en su posición financiera en el mediano y largo plazo; recordemos que cualquier tipo de contaminación es una ineficiencia desde el punto de vista de la utilización de los recursos.

Por otra parte, la intervención de organismos de control externos a la empresa, como lo puede ser una autoridad central, tendría como consecuencia un incremento en los costos de producción de las empresas, situación que en el largo plazo puede impactar en su competitividad y estabilidad financiera. 
La importancia de plasmar estas relaciones a través de un modelo matemático se da a partir de que - a diferencia de otros trabajos en los que se plantean relaciones estadísticas o econométricas en un intento por mostrar la relación entre algunas variables y el comportamiento medioambiental de las empresas (por ejemplo, Al-Tuwaijiri et al., 2004) o trabajos en los que se plantean situaciones que teóricamente debería ocurrir en el comportamiento organizacional con respecto al entorno natural (como Porter y Van der Linde, 1995a y b, y Walley y Whitehead, 1994) - bajo el modelo planteado podemos plasmar la lógica del pensamiento económico en la toma de decisiones de la empresa, entorno en el cual impera la lógica de maximización del beneficio.

En este sentido, a través del modelo desarrollado, es posible apreciar cómo las empresas optarán por implementar — desde la perspectiva de maximización del beneficio y de forma voluntaria o bien por la intervención de una autoridad central como agente de control- políticas amigables con el medioambiente como un esfuerzo por mantener o incrementar los beneficios presentes y futuros (no olvidemos que el modelo incluye el elemento de tasa de descuento intertemporal) de la organización.

El papel que juega el mundo empresarial en la mejora de la calidad de vida de todos es crucial para poder alcanzar lo que dice el concepto de desarrollo sustentable; es decir, poder satisfacer las necesidades de las generaciones presentes sin poner en riesgo la satisfacción de las necesidades de las generaciones futuras, brindándoles a ellos las mismas oportunidades que hemos tenido nosotros al vivir.

\section{Referencias}

Aitken, W. R. O. (1989). Conserving the environment: sustaining economic growth. Taking environment concerns in to accounts makes good business care. Canadian Business Review 16 (2): 17.

Al-Tuwaijri, S. A., T. E. Christensen y K. E. Hughes (2004). The relations among environmental disclosure, environmental performance, and economic performance: a simultaneous equations approach. Accounting, Organizations and Society 29 (5-6): 447-471. 
Amnistia, I. (2002). Empresas más responsables para una Europa más justa. Boletin Economico del ICE 2728: 41-43.

Asociacion Española de Contabilidad y Administración de Empresas (2004). Marco conceptual de la responsabilidad social corporativa. Madrid.

Banerjee, S. B. (2001). Managerial perceptions of corporate environmentalism: intepretations from industry and strategic implications for organizations. Journal of Management Studies 38 (4): 489-513.

Bebbington, J. (2001). Sustainable development: a review of the international development, business and accounting literature. Accounting Forum 25 (2): 128-157.

Carroll, A. (1979). A three dimensional conceptual model of corporate performance. Academy of Management Review 4 (4): 17.

Castellanos, M., I. Martín y F. J. Caro (2007). Propuesta de una escala de medición de la responsabilida social corproativa (RSC) en la actividad turistica. Conocimiento, innovación y emprendedores: camino a futuro. C. p. J. C. A. Calvo, Grupo de Investigación Fedra.

Comisión de las Comunidades Europeas (2001). Libro verde: Fomentar un marco europeo para la responsabilidad social de las empresas. Bruselas.

Covey, S. R. (2003). Los 7 hábitos de la gente altamente efectiva. Buenos Aires: Paidós.

Crespo, C. (2002). Internalización de la variable medioambiental: respuesta estrategica, cambio organizativo e implicacion de los sistemas de información contable. Estudio de un caso multiple. Tesis doctoral, Valencia, España, Universidad de Valencia.

Fernández de Gatta, D. (2004). Auditorías y sistemas de gestión ambientales en la Union Europea: evolución, régimen vigente y perspectivas futuras. Noticias de la Union Europea 228 (Enero). 
Fernández, R. (2005). Administración de la responsabilidad social corporativa. España: Thompson Editores.

Fundación Entorno (2003). Informe sobre gestión medioambiental en la empresa española. Madrid: Entorno.

Gallizo, J. L. (2006). Informar sobre la responsabilidad social de la empresa. Algo más que una moda. Responsabilidad e Información Medioambiental en la Empresa A. E. d. C. y. A. d. E. (AECA): 11-23.

Garcés, C. (2006). Estrategias empresariales de responsabilidad medioambiental: presion de los stakeholders y barreras a la adaptacion. Responsabilidad social e informacion medioambiental de la empresa. A. E. d. C. y. A. d. E. (AECA).

Giraldo, E., A. Durán y A. Durán (2006). Implantación gradual de la responsabilidad social corporativa (RSC) en las empresas: dimensiones de avance y papel de la acreditación progresiva. Revista de Dirección, Organización y Administración de Empresas (32): 37-44.

Gray, R. y J. Bebbington (2000). Environmental accounting, managerialism and sustainability: Is the planet safe in the hands of business and accounting? Advances in Environmental Accounting \& Management 1 (1): 1-44.

Henri, J.-F. y M. Journeault (2009). Eco-control: The influence of management control systems on environmental and economic performance. Accounting, Organizations and Society In Press Corrected Proof.

Hernández, M. C. (1999). Respuesta del sistema informativo contable a la responsabilidad social de la empresa: especial referencia a España. Técnica Contable LI: 375-394.

Hoffmeister, L., F. G. Benavides y P. Jodar (2005). Responsabilidad social corporativa en salud y seguridad en el trabajo: dimensiones, realidad y perspectivas. Cuaderno de relaciones laborales 24 (1): 18 -198.

Lara, M. I. de (2003). La responsabilidad social en la empresa: implicaciones contables. Madrid: Edisofer. 
Larrinaga, C. (2002). Contabilidad y desarrollo sostenible. X encuentro de Profesores Universitarios de Contabilidad, Santiago de Compostela: 1-18.

Lizcano, J. L. y P. Nieto (2006). La responsabilidad social en el capitalismo futuro. Competencia, gasto público y comportamiento social de la empresa. Universia Business Review. Actualidad Economica, segundo trimestre: 10-23.

Moneva, J. M. y F. Llena (2006). Contabilidad e información medioambiental de la empresa. Antecedentes y situacióna actual. Responsabilidad Social e Información Medioambiental en la Empresa. Asociación Española de Contabilidad y Administración de Empresas.

Moreno, J. A. (2004). Responsabilidad social corporativa y competitividad: una visión desde la empresa. Revista Valenciana de Economía y Hacienda (12): $9-50$.

Nieto, M. (2004). ¿Por qué adoptan criterios RSC las empresas españolas? Revista Economistas 104 (Extra España 2004: un balance).

(2005). Difusión de la responsabilidad social corporativa en la empresa española. Revista Economistas 106 (XXII): 32 - 44.

Nieto, M. y R. Fernández (2004). Responsabilidad social corporativa: la última innovación en management. Universia Business Review. Actualidad Económica primer trimestre: 28-39.

Porter, M. E. y C. van der Linde (1995a). Green and Competitive: Ending the Stalemate. Harvard Business Review 73 (5): 120-134.

y C. Van der Linde (1995b). Toward a new conception of the environment-competitiveness relationship. Journal of Economic Perspectives 9 (4): 97-118.

y M. R. Kramer (2003). La filantropía empresarial como ventaja competitiva. Harvard Deusto Business Review enero-febrero (12): 6-20.

UNWCED (1987). Our Common Future (The Brundtland Report). O. O. U. Press. 
Vaca, R. M., M. J. Moreno y F. Riquel (2007). Análisis de la responsabilidad social corporativa desde tres enfoques: stakeholders, capital intelectual y teoría institucional. Conocimiento, innovación y emprendedores: camino a futuro. C. p. J. C. A. Calvo, Grupo de Investigación Fedra.

Walley, N. y B. Whitehead (1994). It's Not Easy Being Green. Harvard Business Review 72 (3): 46-51. 